\title{
A new Particle Swarm Optimization Algorithm based on Periodic
}

\section{Changing Inertia Weight}

\author{
Mingyan Zheng ${ }^{1, a^{*}}$,Siyan Wang ${ }^{1, b}$ and Yan $\mathrm{Li}^{1, \mathrm{c}}$ \\ ${ }^{1}$ Department of Information Engineering, Weifang Business Vocational College \\ Weifang,shandong,China \\ asdzczmy@163.com, bwangsiyan1985@163.com, 'ljxhaoljxhao@126.com
}

Keywords: inertia weight; continuous decline; period; large range search; small range search

\begin{abstract}
The new inertia weight based on periodic linear change, $\omega$ repeat the change process from 0.9226 to 0.6226 until it reaches the maximum generation. At the beginning, the position of the particle change fast, when the given value is reached, the particle's position change with a fuzzy formula. Using the new algorithm, the global and local searching ability of the particle swarm optimization algorithm are improved obviously, the searching accuracy is also improved. To a certain extent, the premature convergence problem of particle swarm algorithm is solved. The algorithm also could be used to solve some complex computational problems in many fields.
\end{abstract}

\section{Introduction}

Particle swarm optimization algorithm is proposed by Dr Eberhart and Kennedy. It originated from the birds preying behavior [1]. In order to control the particle's convergence and search ability, Shi et al introduced inertia weight[2] in the algorithm. Particle swarm optimization algorithm has been successfully applied to calculate complex functions' result, system identification.

In the past, many people had some new ideas to improve the performance of the PSO algorithm, but they often tend to change the operator very complex. So the new algorithm they have proposed often increased the complexity of the PSO algorithm.

This paper presents a new particle swarm optimization algorithm based on Periodic changing Inertia weight (PIPSO), the inertia weight changes with series linear cycle. With the new method, particles can better adjust their flight direction and speed. The results of test functions show that the algorithm is a simple algorithm that with fast convergence speed, and has good convergence precision.

\section{Particle Swarm Optimization Algorithm}

The standard particle swarm algorithm is assumed to contain $\mathrm{m}$ particles in a D dimension space., mainly described in Eq. 1[3], Each particle adjusts their flight speed and direction according to the current speed, current location, personal best solution and the global optimal solution, there's the adjustment formula(Eq. 2 and Eq. 3)[4]. Among Eq. 2 and Eq. $3 i=1,2, \cdots, m ; d=1,2, \cdots, D ; r_{1}, r_{2}$, is random number in the range of $(0,1) ; c_{1}, c_{2}$ are learning factors. 


$$
\begin{aligned}
& \left\{\begin{array}{l}
x_{i}(t)=\left(x_{i 1}(t), x_{i 2}(t), \cdots, x_{i d}(t)\right)(\text { the current } \\
\text { position of thei particle }) \\
v_{i}(t)=\left(x_{i 1}(t), x_{i 2}(t), \cdots, x_{i d}(t)\right)((\text { the current } \\
\text { speed of thei particle })) \\
p b_{i}(t)=\left(p b_{i 1}(t), p b_{i 2}(t), \cdots p b_{i d}(t)\right)(\text { individual } \\
\text { optimalsolution }) \\
\text { gb }(\mathrm{t})=\left(g b_{1}(t), g b_{2}(t), \cdots g b_{\mathrm{d}}(t)\right)(\text { theglobal } \\
\text { optimalsolution })
\end{array}\right. \\
& v_{i d}(t+1)=\omega * v_{i d}(t)+c_{1} * r_{1} *\left(p b_{i d}(t)-x_{i d}(t)\right)+c_{2} * r_{2} *\left(g b(t)-x_{i d}(t)\right) \\
& x_{i d}(t+1)=x_{i d}(t)+v_{i d}(t+1)
\end{aligned}
$$

\section{A new Particle Swarm Optimization Algorithm based on Periodic Changing Inertia Weight}

\subsection{Periodic changing inertia weight is proposed}

The inertia weight $\omega$ plays a key role in the particle swarm algorithm, its size in relation to the whole group of search range[5]. In some paper, although authors have proposed a lot of new method to change inertia weight, but some of them made the inertia weight monotone decreasing or monotone increasing, some other people made the inertia weight first increasing then decreasing, or first decreasing then increasing.

This paper takes advantage of the principle of searching things in our daily life. When someone search something, if he or she cannot find it in one place after a period of time, he or she maybe goes to another place and search it in this place. What is called turning to another place is that through a big mutation probability, obtaining some new individual from a new region that is far from the ancestor of them. The specific operation process is as follows: $\omega$ 's maximum value is 0.9226 , minimum value is 0.6226 , in the beginning, its value is 0.9226 , then it begins to decline until 0.6226 , decreasing unit is 0.003 . $\omega$ repeat the linear change process that from 0.9226 to 0.6226 until it reaches the maximum generation. The change process is shown in Fig. 1.

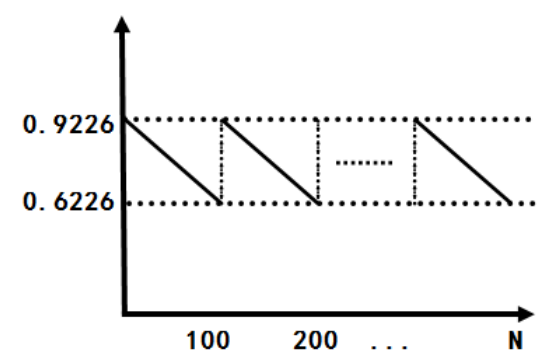

Figure. 1 change process of inertia weight

\subsection{Fuzzy update position by stages}

A new method is used to update the position of the particle, which is "fuzzy update position by stages". When $\omega$ is decreasing, the position of the particle also fuzzy update by stages. In every decreasing stage, when the evolutional generation is relatively small, degree of membership is 1 ; the position of the particle changes consistent with $\mathrm{v}_{\mathrm{id}}(\mathrm{t}+1)$; when the evolutional generation is 
greater than a given threshold, the degree of membership decrease to a given value, the position of the particle changes slowly; When the evolutional generation arrive another given threshold, the degree of membership increase to a new value. The change is very simple, but it can effectively improve the speed of algorithm. Fuzzy control formulas are as follows (Eq. 4 and Eq. 5):

$$
\begin{aligned}
& x_{i d}(t+1)=x_{i d}(t)+\lambda * v_{i d}(t+1) \\
& \lambda= \begin{cases}1 & \left(t \leq T_{1}\right) \\
\lambda_{1} & \left(T_{1}<t \leq T_{2}\right) \\
1 & \left(t>T_{2}\right)\end{cases}
\end{aligned}
$$

Among Eq. 5, $\lambda$ is a piecewise function, $\mathrm{T}_{1}, \mathrm{~T}_{2}$ are given threshold. Instead of using Gaussian, Bell, Sigmoid, Triangle etc membership function, in this paper, the membership function is changing with a periodic linear process. It's simple and feasible, the initial value $\lambda_{1}^{0}$ decreasing unit $\lambda_{1}^{1}$ and the lower limit value $\lambda_{1}^{2}$, all can be flexibly transform and validate according to specific target function. This could save the time for choosing membership function, simplify the calculation process, improve the calculation accuracy significantly and accelerate convergence speed.

\subsection{The new particle swarm optimization algorithm process based on periodic change}

(1)Set the parameter like the evolving algebra, the dimensions of the problem etc. Random initialize of the particle's position and speed [6].

(2)According to the problem needed to be optimized, calculate the fitness of each particle, sort them, thus get the most optimal pbest, gbest is set to the best particle's position of the current group.

(3)Update the inertia weight and the particle's position according to the new calculation method; get the new speed and the position of the particle.

(4)To get a new global optimal value judgment, observe whether meet the accuracy requirement, or whether the iteration algorithm has reached the setup evolving algebra. If yes, the iteration process is over; If not, continue the arithmetic, repeat the step (2)-(4).

\section{The analysis of new particle swarm optimization algorithm based on periodic change}

To verify the performance of the algorithm, contrast test the new particle swarm optimization algorithm based on periodic change and the algorithm of the reference [3]. The test functions' expressions are in Table I:

Table I test function

\begin{tabular}{|c|l|}
\hline FunctionRosenlbrock & $f_{1}(x)=\sum_{i=1}^{n-1}\left[100\left(x_{i+1}-x_{i}\right)^{2}+\left(x_{i}-1\right)^{2}\right]$ \\
\hline FunctionRastrigin & $f_{2}(x)=\sum_{i=1}^{n}\left[x_{i}^{2}-10 \cos \left(2 \pi x_{i}\right)+10\right]$ \\
\hline FunctionGriewank & $f_{3}(x)=\frac{1}{4000} \sum_{i=1}^{n} x_{i}^{2}-\prod_{i=1}^{n} \cos \left(\frac{x_{i}}{\sqrt{i}}\right)+1$ \\
\hline FunctionAckley & $f_{4}(x)=-20 \exp \left(-0.2 \sqrt{\frac{1}{n} \sum_{i=1}^{n} x_{i}^{2}}\right)-\exp \left(\frac{1}{n} \sum_{i=1}^{n} 2 \pi x_{i}+2\right)+20+e$ \\
\hline
\end{tabular}

This experiment is mainly to test the minimum value of the four typical functions. The experiment parameter is set to: the particle population size is 100 , the maximum number of 
evolution is 1000; In PSO, $\omega$ is 0.9226; In WPSO, $\omega$ linear decreases to 0.6 with the increase of evolution algebra; In AFPSO, $\omega$ is fuzzy changed according to the given membership function; In PIPSO, the maximum value is 0.9226 , the minimum value is 0.6226 , $\omega$ starts from 0.9226 to 0.003 monotone decreasing for the unit $\omega$ decreases to 0.6226 , $\omega$ will repeat the process from 0.9226 to 0.6226 until reaching to the maximum evolving algebra. Each function test runs 50 times, their average is as the final result of the algorithm optimization. The average value is shown in Table II.

Table II the minimum value of the four typical functions

\begin{tabular}{lllllll}
\hline Function & Search Limit & Dimension & PSO & WPSO & AFPSO & PIPSO \\
\hline$f_{1}(x)$ & {$[-100,100]$} & {$[-20,20]$} & 3.6241 & 2.7845 & 2.1764 & 1.4213 \\
$f_{2}(x)$ & {$[-100,100]$} & {$[-30,30]$} & 7.4234 & 6.7821 & 6.2735 & 4.7127 \\
$f_{3}(x)$ & {$[-512,512]$} & {$[-20,20]$} & 6.1381 & 5.4472 & 5.0846 & 4.1235 \\
$f_{4}(x)$ & {$[-512,512]$} & {$[-30,30]$} & 6.2383 & 4.3481 & 4.1472 & 3.2156 \\
\hline
\end{tabular}

As shown in Table II, the PIPSO algorithm referenced in this file, has improved the performance greatly than that of the other three algorithms, minimum value is more close to 0 , which is the typical function theory of optimal value.

Fig. 2 is the iterative process's tracking of four typical test functions, through the relation schema between the four typical function algebra's evolving algebra and the average fitness of the best diagram, using the PIPSO, Individual toward the optimal position moving faster and it could effectively avoid the algorithm into local optimum during the initial stage and final stage, this could demonstrate the superiority of the fuzzy position update. At the same time, we could also find out that in the process of particle movement, there will be a step towards the optimal position of the larger mobile long, this greatly accelerate the convergence speed of the algorithm and improve the convergence precision.

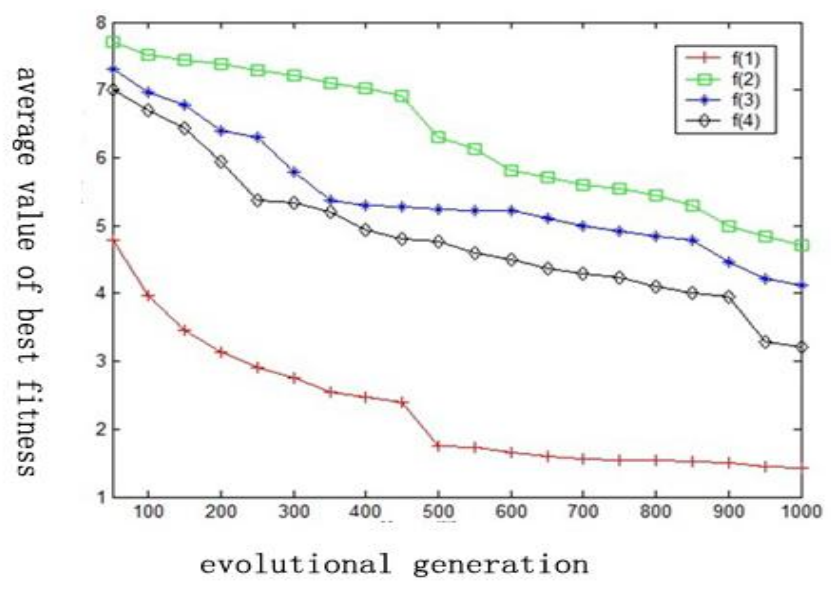

Figure. 2 iterative process's tracking of four typical test functions

\section{Conclusions}

Present a particle swarm optimization algorithm-PIPSO, Propose simulation 'losing things looking for things' of inertia weight change method and the particle position fuzzy updating method, through the study of the test of typical function, PIPSO improve the convergence precision more than other algorithm, and it avoid algorithm trapped in local optimum.

\section{Acknowledgment}

This work was supported by the Research Department of Weifang Business Vocational College. 
Thanks for the support of our school.

\section{References}

[1] J.Kennedy, R.C.Eberhart . Particle swarm optimization [A] . Proc IEEE international conference on Neural Networks [ C ] . USA: IEEE Press,(1995), P. 1942- 1948.

[2] Y Shi, R C Eberhart . A modified swarm optimizer, in IEEE International Conference of Evolut ionary Computation[ C] .Anchorage, Alaska:IEEE Press,May, (1998).

[3] Songhua ZHOU,Chunjuan OUYANG,Changxin LIU,Ping zhu, in:Computer Engineering and Applications. 46-48(2009) P.47.

[4]R.C.Eberhart, J.Kennedy, A new optimizer using particle swarm theory, in: Proceedings of the Sixth International Symposium on Micro Machines and Human Science,(1995), P.39-43.

[5] V.Vaidehi, Bhairavi Kannan,Harini Murugan. Hierarchical Particle Swarm Optimization with Ortho-Cyclin Circles, in:Expert Systems with Applications 41(2014) P.3460-3476.

[6] Zhensu Lv,Zhirong Hou.Particle Swarm Optimization with Adaptive Mutation, in:ACTA ELECTRONICA SINCA. Vol.32 No.3 Mar.(2004). 